\title{
Bacteriological Profile of Early and Late onset Ventilator Associated Pneumonia
}

\author{
S. Sadiqa Begum ${ }^{1} *$, Mariraj Jeer ${ }^{2}$ and S. Krishna ${ }^{2}$ \\ ${ }^{1}$ Department of Microbiology, Subbayya Institute of Medical Sciences, \\ Shimoga-577201, India \\ ${ }^{2}$ Vijayanagara Institute of Medical Sciences, Ballari-583101, India \\ *Corresponding author
}

\section{Keywords}

VAP, Mechanical ventilation, MDR,

Endotracheal aspirate (ETA)

Article Info

Accepted:

25 February 2020

Available Online:

10 March 2020

\section{A B S T R A C T}

Ventilator-associated pneumonia is a common complication in intensive care units, occurring in $9 \%$ to $24 \%$ of patients intubated for longer than 48 hours. This study aims to determine bacteriological profile of early and late onset VAP and its antibiotic susceptibility pattern. A prospective study was performed in the ICU setting of a tertiary care hospital (VIMS, Ballari) over a period of 1 year. ETA culture of 68 patients with a clinical suspicion of VAP were analysed by standard microbiological procedures. The present study showed GNB as the most common organism isolated. Pseudomonas spp (24.6\%), followed by Klebsiella spp (22.8\%), Acenitobacter spp (12.3\%), former being predominant in early onset variety \& the latter in the late onset VAP. Other organisms like E.coli, Citrobacter species, Proteus species, staphylococcus aureus and CONS are isolated in their decreasing frequency. Most of the organisms are resistance to Beta lactams and cephalosporins but sensitive to carbepenems. The Bacteriological culture of endobroncheal aspirates is helpful in the diagnosis and management of VAP. MDR is seen in most patients of VAP, Carbepenems are effective in treatment.

\section{Introduction}

HAP and VAP represented the second most common nosocomial infection affecting approximately $27 \%$ of all critically ill patients ${ }^{1}$.Ventilator-associated pneumonia is a common complication in intensive care units, occurring in $9 \%$ to $24 \%$ of patients intubated for longer than 48 hours $^{2}$. Ventilator- associated pneumonia (VAP) is defined as pneumonia occurring in a mechanically ventilated patient after 48 hours of endotracheal intubation ${ }^{3}$.Microorganisms responsible for VAP may differ according to the population of patients in the ICU, the durations of hospital and ICU stays, and the specific diagnostic methods used ${ }^{4}$.Major bacterial isolates from HAP and VAP cases in 
Asian countries were Acinetobacter spp, Pseudomonas aeruginosa, Staphylococcus aureus and Klebsiella pneumoniae ${ }^{5}$.

The spectrum of potential pathogens can be defined by assessment of a variety of factors, including the severity of the pneumonia itself, the presence of specific co-existing illness, prior therapy (including antibiotics), and length of hospitalization ${ }^{6}$. Quantitative endotracheal aspirate culture is a useful noninvasive tool for the diagnosis of pneumonia pathogens in critically ill patients, findings suggest that a threshold level of $10^{6} \mathrm{cfu} / \mathrm{mL}$ is appropriate because at this level excellent concordance was found with quantitative BAL culture results ${ }^{7}$. So the aim of the study is to determine bacteriological profile of early onset and late onset VAP from ETA samples and its antibiotic susceptibility pattern for the optimal treatment of patients and to control the spread of resistance.

\section{Materials and Methods}

A prospective study done enrolling patients, from the ICU of our hospital, VIMS Ballari for a period of One year (From January 2015 to December 2015). Intubated patients (>18 years of age) who are mechanically ventilated for more than 48 hours, with a clinical suspicion of ventilator-associated pneumonia, patients with two of the following:Fever $\geq 38$ ${ }^{\circ} \mathrm{C}$, Purulent Bronchial Secretions, and WBC more than $10,000 / \mathrm{mm}^{3}$ or $<3000 / \mathrm{mm}^{3}$ were included in the study. Patients with pneumonia or any pre-existing lower respiratory tract infections at the time of admission or first $48 \mathrm{hrs}$ of mechanical ventilation were excluded in this study. Clinically diagnosed Ventilator associated pneumonia cases were observed and data such as name, age, gender, date of admission into intensive care unit, chief complaints, risk factors involved, duration of mechanical ventilation were obtained. Data related to general physical examination, radiological and haematological investigations was also collected.

Endotracheal aspirate (ETA) was collected under aseptic precautions. A sterile 22 inches suction catheter (Ramsons) was introduced into respiratory tract for a distance of 20$25 \mathrm{cms}$ and the specimen was aspirated into a sterile container. The sample was transported to the laboratory immediately for processing.

The ETA so collected was subjected to Gram's stain (Microscopy). The specimen was further diluted using sterile saline (1 in 100) and the dilution factor was noted and the specimen was then inoculated for quantitative culture using standard techniques onto MacConkey agar, Blood agar and Chocolate agar plates using a $4 \mathrm{~mm}$ sterile nichrome loop $(0.01 \mathrm{ml})$, and then incubated at $37^{\circ} \mathrm{C}$. The plates were examined for growth at $24 \mathrm{hrs}$, and if there was no growth, it was further incubated for another $24 \mathrm{hrs}$. The CFU/ml was then calculated, considering the dilution factor.

$\mathrm{CFU} / \mathrm{ml}=$ No. of colonies grown $\mathrm{x}$ Dilution Factor $\left(10^{2}\right) \times$ Reciprocal of volume $(\mathrm{ml})$ of specimen used for inoculation $\left(10^{2}\right)$.

Bacterial growth with $>10^{6} \mathrm{CFU} / \mathrm{ml}$ was given significance as a pathogen and was further identified using appropriate biochemical tests. The antibiotic susceptibility testing was performed according to CLSI guidelines.

\section{Results and Discussion}

A prospective study was done enrolling 68 patients out of 240 patients on mechanical ventilation as per the inclusion criteria, and the following results were obtained. Of the total 68 patients suspected to have VAP, 52 (76.5\%) developed true VAP as per the diagnostic criteria. Incidence rate of VAP in 
our study is $21.7 \%$. The significant peak of incidence is seen in 25-45yrs of age group. Among these $46(67.6 \%)$ were male and 22 $(32.4 \%)$ were females..Late onset pneumonia was suspected in $31(59.6 \%)$ cases and early onset pneumonia in 21(40.4\%) cases. Out of 52 cases of true VAP, 24(46\%) were admitted for Surgical problems, $16(30 \%)$ for medical problems and 12(23\%) for others. Total mortality rate was $21 \%$

Out of the 52 cases, 47(90.4\%) had monomicrobial bacterial growth and 5(9.6\%) cases showed polymicrobial growth and $16(23.5 \%)$ cases had no significant growth on culture. $70.2 \%$ of Gram negative bacteria and $29.8 \%$ of Gram positive isolates were isolated. The predominant GNB isolated in our study is Pseudomonas spp (24.6\%), followed by KlebsiellaSpp (22.8\%), AcenitobacterSpp (12.3\%), CitrobacterSpp (7.2\%), E.coli (1.7\%), Proteus Spp (1.7\%)(Graph 1). Amongst the Gram positive isolates Staphylococcus aureus (22.8\%) was most frequently isolated. $70 \%$ of GNB are ESBL producer and $10(77 \%)$ are MRSA and $3(22 \%)$ are MSSA.

VAP is the most prevalent nosocomial infection, leading to increased hospital stay, high cost, morbidity and mortality.In different studies, the incidence of VAP was reported different; depending on the type of hospital or ICU, the population studied and the organisms prevalent; and varied from $7 \%$ to $70 \%$. This study was conducted to assess the bacterial etiological agents leading to early and late onset VAP.

Our study showed occurrence of VAP to be $21.7 \%$, which was in close agreement with a study conducted on Nosocomial pneumonia by Vincent JL $(2001)^{8}$. In a South Indian study, Rakshith P. et al., ${ }^{9}(2005)$ reported the incidence to be 26 per 1000 ventilator days.

The patients studied belonged predominantly to the age group of 25-45 years with a significant peak incidence of 42.6 similar to $40-49 y r s$ in a study by Nigeria by V. Olugbue $^{10}(2011)$. Late onset VAP $(59.6 \%)$ was predominant in our study. The more the number of days the patient is on a ventilator, the higher is chance of getting pneumonia. This has been proved beyond doubt by Sanmath K et al., ${ }^{11}$ where90.32\% developed Late onset VAP and $34.83 \%$ developed early VAP. In the present study, Surgical conditions -post-operative procedures $(46 \%)$ is the most common risk factor noted followed by medical conditions $(30.7 \%)$.

In our study, the incidence of aerobic gram negative bacteria is $70.2 \%$ and this high incidence correlated with other studies like Kanj SS et al., ${ }^{12}$ which showed incidence of GNB of $83 \%$. Johanson et al., isolated 22 isolates of GNB from 26 patients with nosocomial respiratory infection ${ }^{13}$.

In a study of Dr.HinaGadani et al., ${ }^{14}$, The order of prevalence of organism in VAP cases was found to be Pseudomonas (43.2\%), Klebsiella (18.91\%), followed by MRSA, Escherichia coli, Acinetobacter, Methicillin Sensitive Staphylococcus aureus (MSSA) and Streptococcus pneumonia which is similar to our study.

In early onset VAP, the organism most commonly isolated was Pseudomonas spp accounting for $30.4 \%$ of all isolates. Klebsiellaspp (23.5\%) followed by Acinetobacter spp (20.6\%) were the most common organism isolated in Late VAP(Table $1)$. 
Table.1 Frequency of organism distribution in Early \& Late VAP

\begin{tabular}{|c|l|c|c|c|c|}
\hline S. No & \multicolumn{1}{|c|}{ Early VAP } & \multicolumn{2}{c|}{ Late VAP } \\
\hline & \multicolumn{1}{|c|}{ Isolate } & No=23 & $\%$ & No=34 & $\%$ \\
\hline $\mathbf{1 .}$ & Pseudomonas $s p p$ & 7 & 30.4 & 7 & 20.9 \\
\hline $\mathbf{2 .}$ & Klebsiella spp & 5 & 21.7 & 8 & 23.5 \\
\hline & K. oxytoca & 5 & 21.7 & 6 & 27.6 \\
\hline & K.pneumoniae & Nil & & 2 & 5.9 \\
\hline $\mathbf{3 .}$ & $\begin{array}{l}\text { Staphylococcus } \\
\text { aureus }\end{array}$ & 6 & 26.1 & 7 & 20.6 \\
\hline $\mathbf{4 .}$ & Acinetobacter spp & Nil & - & 7 & 20.6 \\
\hline $\mathbf{5 .}$ & Citrobacter koseri & 2 & 8.7 & 2 & 5.9 \\
\hline $\mathbf{6 .}$ & CONS & 2 & 8.7 & 2 & 5.9 \\
\hline $\mathbf{7 .}$ & Proteus mirabilis & 1 & 4.3 & Nil & \\
\hline $\mathbf{8 .}$ & E.coli & 1 & 4.3 & Nil & \\
\hline
\end{tabular}

Among the isolated organism Non fermenter i.e Pseudomonas spp $(30.4 \%)$ is most common causative agent in Early VAP. Klebsiella oxytoca (27.6\%) is most common organism isolated in Late VAP.

Graph.1 Organism isolated

\section{ORGANISMS ISOLATED}

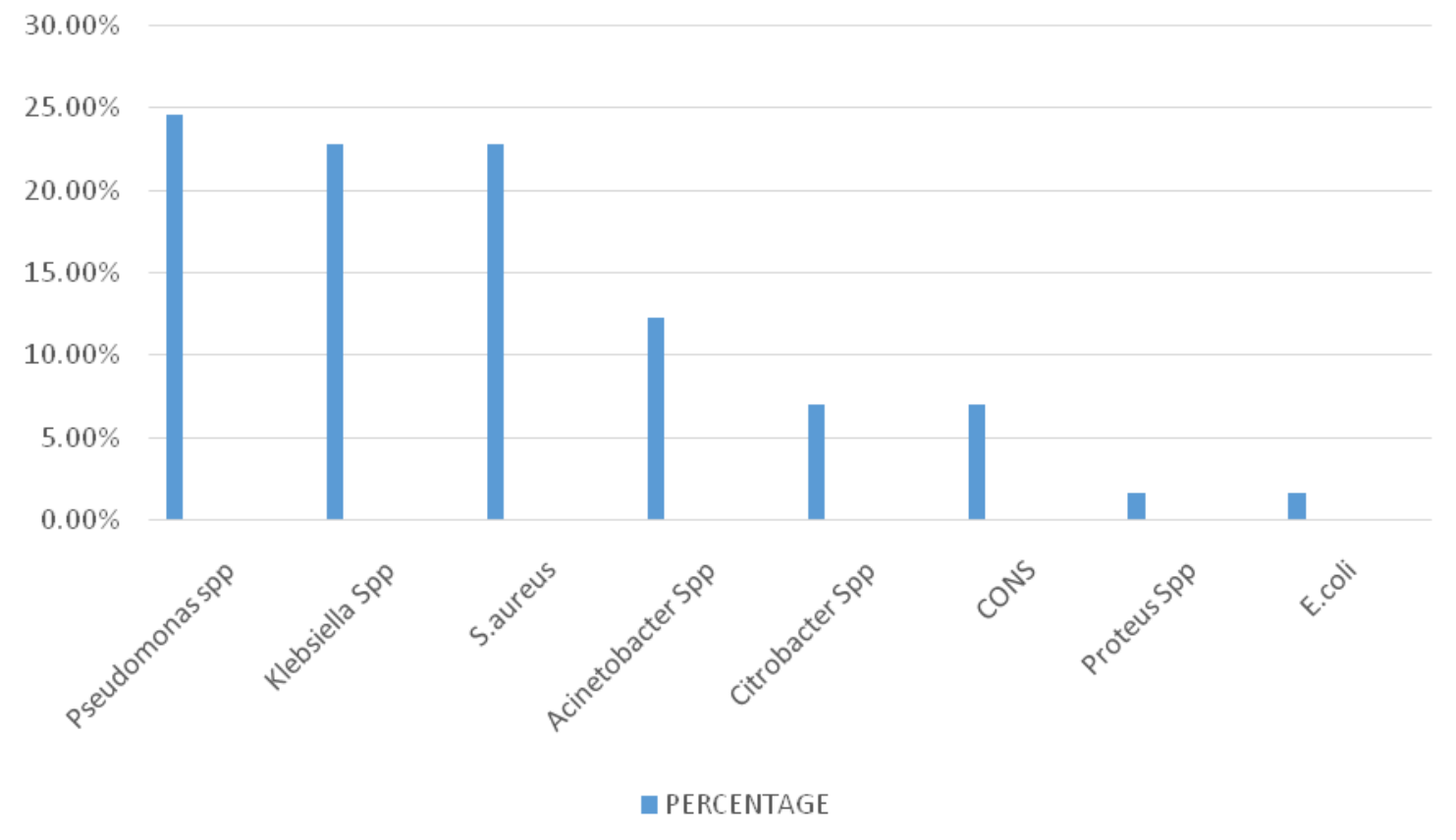

Pseudomonas $(24.6 \%)$ was the most common isolate that was identified. The other significant Gram negative isolates implicated were, Klebsiella spp, Acenitobacter baumanii, E.coli, Proteus spp. Amongst the Gram positive isolates Staphylococcus aureus (22.8\%) was most frequently isolated. 
Fig.1 Pseudomonas aeruginosa on Nutrient agar

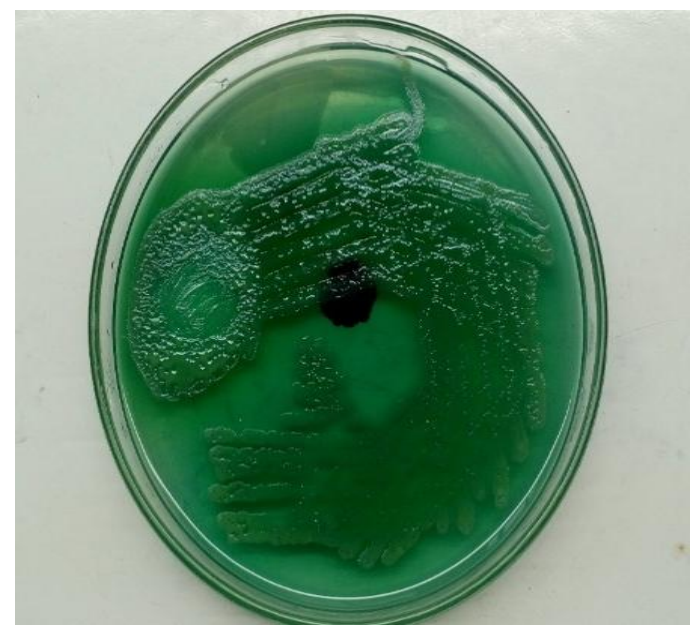

This study correlates with other studies. Ibrahim and co-workers found that the most common pathogen associated with early-onset VAP was Pseudomonas aeruginosa $(25 \%)^{15}$. Rajasekhar et al., (2006) in their study found that, the predominant organisms in the early onset VAP group were Acinetobacter and Klebsiella. In the late onset group Pseudomonas was the most predominant organism.

Nidhi goel et al., study shows $82.4 \%$ of single organism and $11.0 \%$ of polymicrobial growth, similar to our study showing $90.4 \%$ monobacterial and $9.6 \%$ polymicrobial isolates ${ }^{17}$.

The organisms responsible for VAP vary according to case mix, institution, prior antibiotic exposure, local resistance patterns, length of mechanical ventilation, and specific diagnostic method(s) used.

Rajasekhar et al., in 2006 studied the role of quantitative cultures of non - bronchoscopic samples in VAP. They concluded that a culture of lower respiratory tract samples obtained by non bronchoscopic method is a good alternative to bronchoscopic samples in the bacteriological diagnosis of $\mathrm{VAP}^{16}$. Most of the organisms are resistance to Beta lactams and cephalosporins but sensitive to carbepenems. This prospective study has addressed the incidence and microbiology of VAP in our tertiary care center. Bacterial pathogens most frequently isolated are Pseudomonas spp, followed by Klebsiella spp, Acenitobacter spp, Staphylococcus aureus, Citrobacter spp, E.coli, Proteus spp in their decreasing order. In early onset VAP, the organism most common in our study was Pseudomonas spp accounting for $30.4 \%$ of all isolates. Klebsiella spp (23.5\%) followed by Acinetobacter spp (20.6\%) were the most common organism isolated in Late VAP.

Culture of ET aspirate is easy, cost-effective procedure which helps in identifying the organism. Thus the microbiological profile \& sensitivity pattern of the local community helps in framing the appropriate institutional antibiotic policy for better outcomes.

\section{References}

Campbell GD, Niederman MS, Broughton MA, Craven DE, Fein AM, fink

Doo Ryeon Chung, Jae-Hoon Song et al., High Prevalence of Multidrug-Resistant Nonfermenters in Hospital-acquired Pneumonia in Asia. Am J RespirCrit Care Med2011;184:1409-1417. 
Girish L. Dandagi. Nosocomial pneumonia in critically ill patients. Lung India. 2010 JulSep;27(3):149-153.

HinaGadani, Arun Vyas, Akhya Kumar Kar et al., A study of ventilator associated pneumonia: incidence, outcome, risk factors and measure to be taken for prevention. Indian journal of anaesthesia. 2010 Nov-Dec; 54(6):535-540.

Ibrahim EH, Ward S, Sherman RN, Kollef MH. A comparative analysis of patient with early-onset vs late-onset nosocomial pneumonia in the ICU setting. Chest. 2000;117:1434-1442.

Jean Chastre and Jean-Yves Fagon. Ventilatorassociated Pneumonia. Am J RespirCrit Care Med. 2002; 165:867-903.

Johansen et al., Nosocomial respiratory infections with gram negative bacilli. Annals of internal medicine. 1992; 77:701706.

Kanj SS, Kanafani ZA et al.,. International Nosocomial infection control consortium findings of device associated infections rate in an intensive care unit of a Lebanese university hospital. J Global Infect Dis. 2012;4:15-21.

Morehead RS, Pinto SJ. Ventilator-associated pneumonia. Arch intern Med. 2000;160:1926-36.

MP et al., American Thoracic Society. Hospitalacquired pneumonia in adults: diagnosis, assessment of severity, initial antimicrobial therapy, and preventive strategies. A consensus statement. Am J RespirCrit Care Med.1995; 153: 17111725.

Nidhi Goel, Uma Chaudhary, Ritu Aggarwal, Kiran Bala. Antibiotic sensitivity pattern of gram negative bacilli isolated from the lower respiratory tract of ventilated patients in the intensive care unit. Indian $\mathbf{J}$ Crit Care Med 2009;13:148-151.

OlugbueV,Onuoha S. Prevalence and antibiotic sensitivity of bacterial agents involved in lower respiratory tract infections. Int. J.Biol.Chem.Sci. 2011 April; 5(2):774781.

PanwarRakshit P, Nagar VS, Deshpande AK. Incidence, clinical outcome and risk stratification of ventilator-associated pneumonia: a prospective cohort study. Indian J Crit Care Med. 2005;9:211-6.

Rajasekhar T, Anuradha K, Suhasini T, Lakshmi $\mathrm{V}$. The role of quantitative cultures of nonbronchoscopic samples in ventilator associated pneumonia. Indian $\mathrm{J}$ Med Microbiol. 2006;24:107-13.

Sanmath K Shetty et al., A Study on risk factors and Microbial profile of Ventilator associated Pneumonia in Intensive care units of a Tertiary care centre. J Basic Appl Med Sci. 2015;2(2):23- 29.

Shin YM, et al.,Usefulness of Quantitative Endotracheal Aspirate Cultures in Intensive Care Unit Patients with Suspected Pneumonia. J Korean Med Sci. 2011; 26:865-869.

Thomas M File, Jr. Recommendations for Treatment of Hospital Acquired and Ventilator-Associated Pneumonia: Review of Recent International Guidelines. Clinical Infectious Diseases. 2010;51(S1):S42-S47.

Zolfaghari PS, Wyncoll DLA. The tracheal tube: gateway to ventilator-associated pneumonia. Critical Care.2011;15:310.

\section{How to cite this article:}

Sadiqa Begum, S., Mariraj Jeer and Krishna, S. 2020. Bacteriological Profile of Early and Lateonset Ventilator Associated Pneumonia. Int.J.Curr.Microbiol.App.Sci. 9(03): 3151-3162. doi: https://doi.org/10.20546/ijcmas.2020.903.361 